Radical nephrectomy (RN) in the treatment of renal cell carcinoma remains the "gold standard", but sparing techniques (nephron sparing surgery, NSS), consisting of enucleation, partial nephrectomy or ex vivo resection, are increasingly gaining in importance. The techniques allow the achievement of better results, with a low rate of complications and mortality. Ex vivo resection is a technique which is becoming equivalent to radical nephrectomy. The risk of multifocal cancer ranges from 6.5 to $28 \%$, whereas when the tumour has a diameter not exceeding $4 \mathrm{~cm}$, the risk of multiplicity drops to $5 \%$. The main risk involved in NSS is the possibility of recurrence in the ipsilateral kidney. The incidence of recurrence ranges between 0 and $10 \%$ and it is the lowest when the tumour has a low malignant potential and does not exceed $4 \mathrm{~cm}$ in diameter. Sometimes, due to the lack of the other kidney, this is the only opportunity that allows preservation of function of the remaining kidney.

The paper describes a patient with a single kidney, affected by a tumour with a diameter of more than $5 \mathrm{~cm}$, reaching the pyelocalyceal structure. Due to infiltration of the renal calyx, the ex vivo technique was adopted, allowing appropriate resection with an oncologically adequate margin and accurate supply of the urinary tract. The technique of surgery and the outcome of treatment in the very few cases described so far are discussed.

Key words: renal carcinoma, partial resection, autotransplantation.

\section{8-year follow-up after partial ex vivo resection of tumour in a single kidney followed by autotransplantation}

\author{
Marek Karczewski ${ }^{1}$, Wojciech Turkiewicz' ${ }^{1}$ Janusz Skowronek², \\ Jacek Karczewski ${ }^{3}$
}

${ }^{1}$ Transplantology and Surgery Unit of the Provincial Hospital in Poznań 2Brachytherapy Department, Wielkopolska Oncology Centre in Poznań ${ }^{3}$ Department of Biology and Environmental Protection of Poznan University of Medical Sciences

\section{Introduction}

Clear cell renal carcinoma accounts for $90 \%$ of all cases of kidney cancer and $2-3 \%$ of all malignancies [1]. It is usually diagnosed during the sixth and seventh decade of life, although the mean age of diagnosis is gradually becoming lower. Radical nephrectomy $(R N)$ in the treatment of clear cell renal carcinoma has ceased to be the "gold standard" for T1a renal tumours and in some cases of stage T1b tumours, where it has been replaced by nephron sparing surgery (NSS) techniques consisting of enucleation, partial nephrectomy or ex vivo resection [2,3]. The main risk involved in NSS techniques is the possibility of cancer recurrence in the ipsilateral kidney because of incomplete resection of malignant tissue or multifocality of lesions. The risk of multifocal cancer forms is $10-20 \%$ [4].

NSS techniques allow the achievement of better results, with a low rate of complications and mortality. For stage T1a and T1b tumours with a diameter below $4 \mathrm{~cm}$, and in selected cases also below $7 \mathrm{~cm}$, the incidence of local and general recurrence does not demonstrate statistical differences between RN and NSS [5, 6]. Also, the survival rates of patients undergoing partial nephrectomy compared to radical nephrectomy are not lower for technically feasible interventions on tumours with a diameter below $7 \mathrm{~cm}$ [7]. In the absence of technical possibilities for in situ resection of tumours exceeding $5 \mathrm{~cm}$ in diameter, the only option left is ex vivo resection followed by kidney autotransplantation. This type of surgery is much more technically challenging and more risky to the patient, but for selected cases it may be the only alternative to $\mathrm{RN}$.

The case reported in this article involves a patient with a single kidney, affected by a tumour with a diameter of more than $5 \mathrm{~cm}$, reaching the pyelocalyceal structure. Because of infiltration of the renal calyces, the ex vivo technique was adopted, allowing appropriate resection with oncologically adequate margins and accurate supply of the urinary tract.

\section{Case description}

The kidney tumour was diagnosed in a patient, aged 48, by ultrasound imaging and then confirmed by computed tomography (CT). The patient had previously undergone nephrectomy of the left kidney due to staghorn lithiasis and pyelonephritis.

$\mathrm{CT}$ of the abdomen was performed preoperatively, revealing incomplete rotation of the right kidney and, in the dorsal part of the kidney, a solid mass, relatively well circumscribed, measuring $55 \times 48 \mathrm{~mm}$, contrast enhanced after the administration of contrast material. The tumour was not found to infil- 
trate the renal hilus. Slight dilatation of the pyelocalyceal system was noted, along with thickening of the posterior (Gerota's) layer of the renal fascia. No abdominal metastases were found. The status post nephrectomy on the left side was evidenced (Fig. 1).

On admission, the concentration of urea and creatinine in blood serum were (UN) $36 \mathrm{mg} / \mathrm{dl}$ and (Cr) $1.2 \mathrm{mg} / \mathrm{dl}$, respectively.

\section{Treatment method}

The procedure was performed using a Johnson \& Johnson Ethicon Endo-Surgery Harmonic Scalpe ${ }^{\circledR}$, an ultrasonically activated scalpel with three basic qualities of action: coaptation, coagulation and cavitation. The scalpel has a minimum thermal effect on the tissues subjected to cutting and coagulation, making it possible to avoid tissue carbonization and incrustation.

The kidney was removed transperitoneally together with the renal capsule, perirenal tissue and lymph nodes. Then, on the table, following perfusion with cold HTK solution, the tumour was removed with an ultrasonic scalpel, with a margin reaching the renal collecting system (Fig. 2). The pyelocalyceal system and renal parenchyma were then reconstructed via suturing and tissue glue. After cooling and reconstruction, the patient's own kidney was transplanted back in the right iliac fossa by performing an anastomosis of three renal arteries and a single renal vein (Fig. 3) with external iliac vessels, and the ureter with urinary bladder with single sutures on a pigtail catheter (Fig. 4). The duration of warm ischaemia was 45 minutes. Acute tubular necrosis (ATN) occurred postoperatively. The patient required a total of nine haemodialysis procedures in the postoperative period. Packed RBC were transfused and erythropoietin (Eprex ${ }^{\circledR}$ ) was administered, together with an antibiotic (cephalosporin) for protective effect.

The subsequent histopathology examination revealed the following findings: Clear cell renal carcinoma. No infiltration of the renal hilus. Infiltration into Gerota's posterior fascia. According to the 2009 classification, the histopathological tumour grade was determined as grade $3-\mathrm{T} 3$ NO MO (Figs. 5 and 6).

A follow-up CT examination performed 7 days postoperatively demonstrated a post-resection scar in the central area. No pathological enhancement or focal lesions were visualized. Retroperitoneal lymph nodes were not enlarged (Fig. 7).

Angio-CT examination of the transplanted kidney performed 2 weeks postoperatively showed the status after wedge resection (i.e. complete resection) of the tumour with a minor infarcted focus within the superior extremity of the kidney on the medial side. Other observations included moderate dilatation of the renal collecting system, three renal arteries anastomosed to the external iliac artery, and two arteries running medially (both $3 \mathrm{~mm}$ in diameter), demonstrating moderate lumen narrowing in the initial sections. The third artery, with a lateral course, the widest $(4 \mathrm{~mm}$ in diameter), had no visible stenosis. Upon removal of the pigtail catheter, 4 weeks postoperatively, the patient was discharged home.

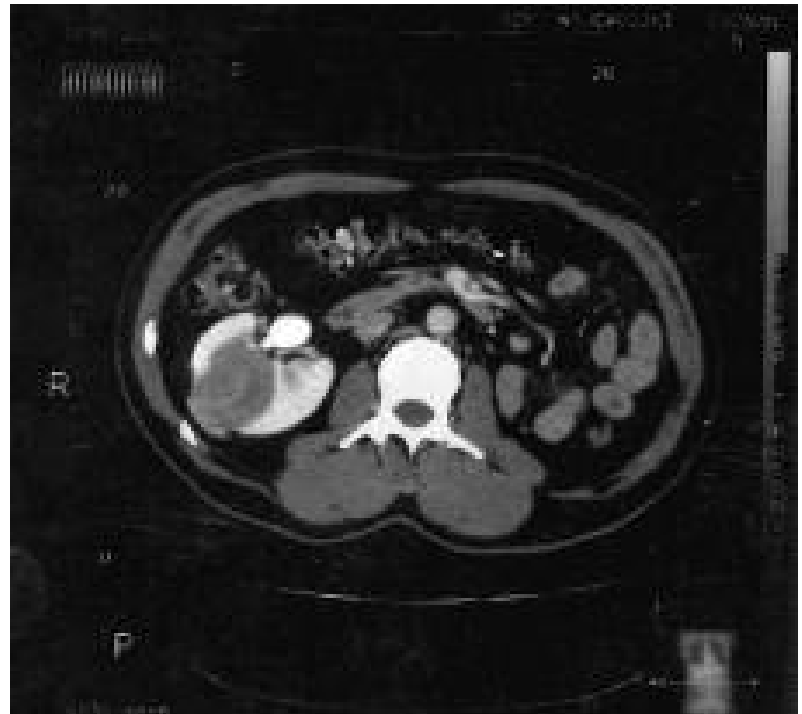

Fig. 1. Solid mass measuring $55 \times 48 \mathrm{~mm}$, relatively well circumscribed, visible in the dorsal part of the right kidney and contrast enhanced after the administration of contrast material.

The tumour does not infiltrate the root of the kidney. Mild dilatation of the pyelocalyceal system

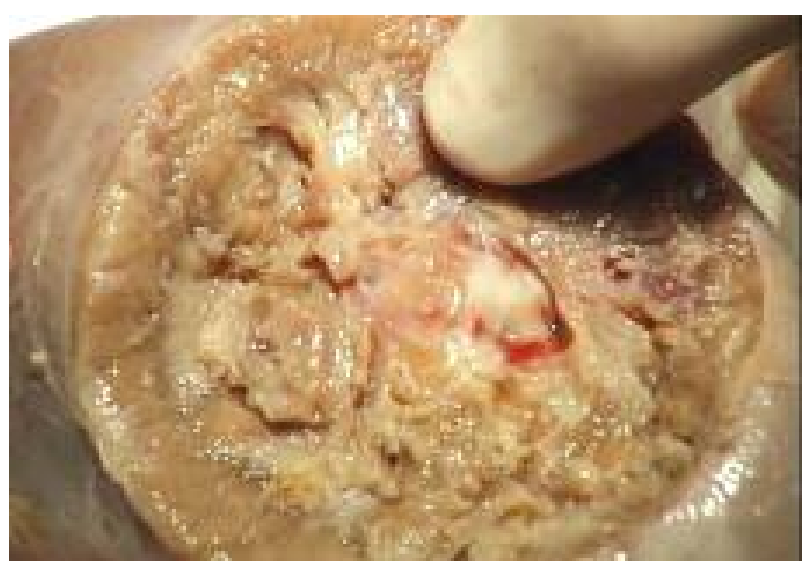

Fig. 2. View post tumour resection with visible penetration into the pyelocalyceal system

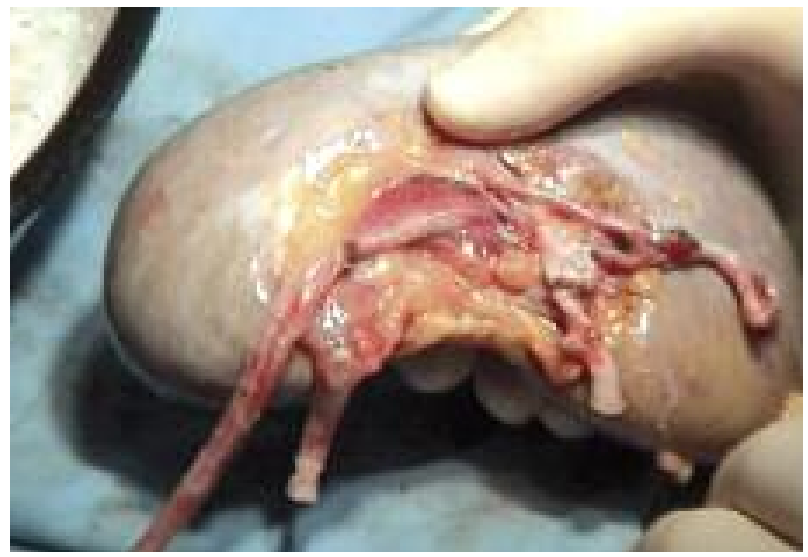

Fig. 3. Kidney with three renal arteries and single renal vein. Two arteries in the upper part of the kidney were first anastomosed side-to-side and then anastomosed in two separate positions to the side of the external iliac vessels 


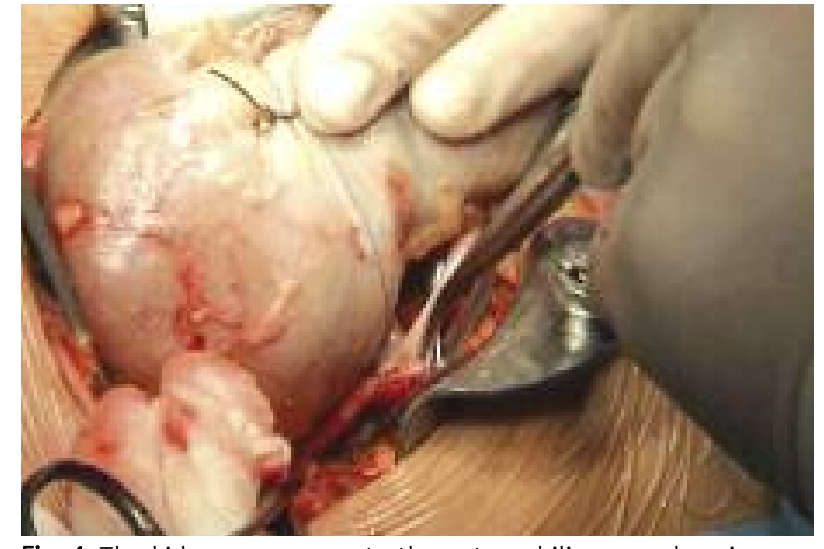

Fig. 4. The kidney was sewn to the external iliac vessels using semicontinuous suture technique. The ureter was fixed to the urinary bladder with interrupted sutures. View showing tumour resection site in the dorsal part of the kidney with the pyelocalyceal system secured by sutures and sealed with tissue glue

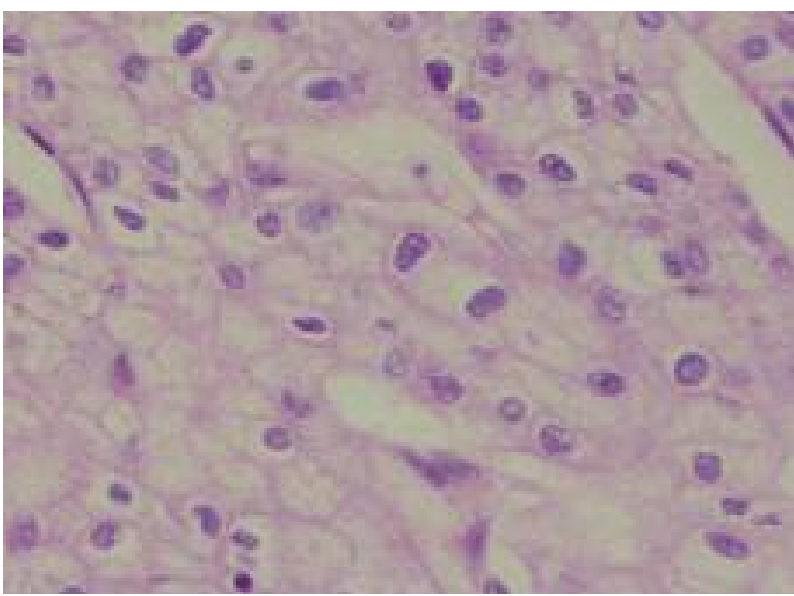

Fig. 6. Histological pattern of clear cell renal carcinoma. Clearly visible cellular membranes. Marked hyperchromasia of part of the nuclei. H\&E stain. Magnification 950x

Table 1. Haemoglobin (HGB), white blood cell (WBC), haematocrit (HTC), urea (UN) and creatinine (Cr) levels

\begin{tabular}{|cccccc|}
\hline & HGB (g/dl) & WBC & HTC & UN & Cr \\
\hline 2002 & 11.7 & 6.6 & 35.2 & 41 & 1.5 \\
\hline 2003 & 11.8 & 6.8 & 41.3 & 43 & 2.3 \\
\hline 2004 & 12.3 & 7.1 & 42.1 & 40 & 2.4 \\
\hline 2005 & 13.6 & 6.5 & 39.9 & 54 & 1.9 \\
\hline 2006 & 14.2 & 6.8 & 42.8 & 67 & 2.1 \\
\hline 2007 & 14.6 & 7.8 & 40.8 & 91 & 2.4 \\
\hline 2008 & 14.7 & 6.3 & 39.5 & 82 & 2.3 \\
\hline 2009 & 14.5 & 6.2 & 39.3 & 80 & 2.4 \\
\hline
\end{tabular}

Six weeks postoperatively the patient was rehospitalized due to hydronephrotic dilatation of the pyelocalyceal system and dysfunction of the transplanted kidney accompanied by elevated serum concentrations of creatinine $(7.0 \mathrm{mg} / \mathrm{dl})$ and urea $(142.3 \mathrm{mg} / \mathrm{dl})$. A revision of the retroperitoneal space revealed a hard cartilaginous infiltration at the site of the ureter-bladder anastomosis. The ureter

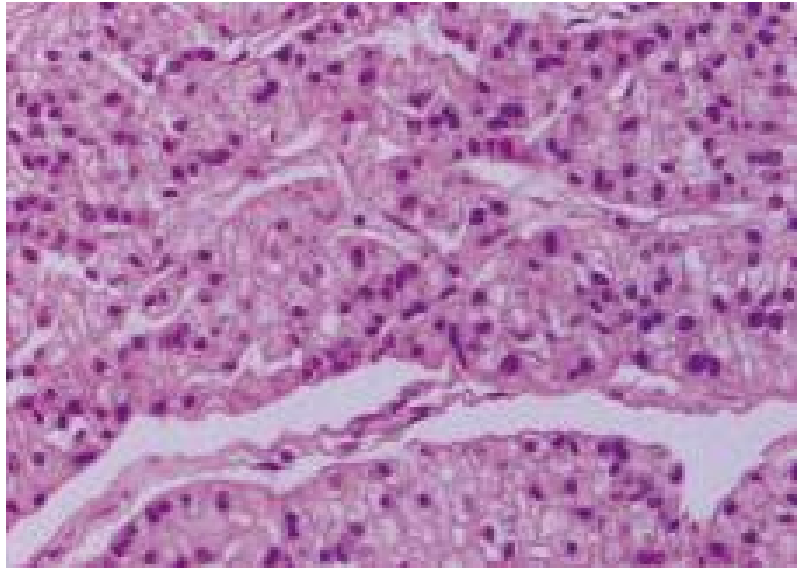

Fig. 5. Histological pattern of clear cell renal carcinoma. Wide vascular lumens are visible. Moderately pronounced variation in the appearance of nuclei and nuclear hyperchromasia. H\&E stain. Magnification 500x

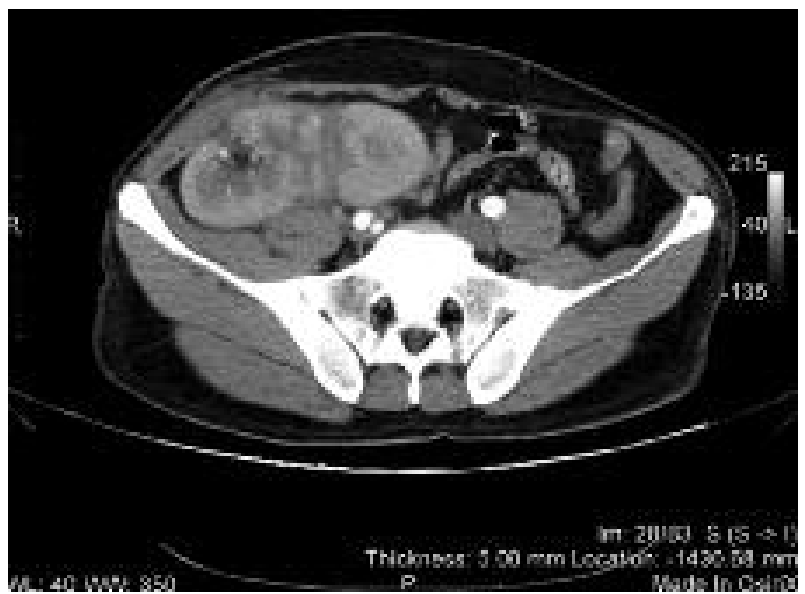

Fig. 7. Follow-up CT examination demonstrating central postresection scar. No pathological enhancement or focal lesions were visualized. Retroperitoneal lymph nodes unenlarged

was reimplanted in the bladder with single sutures on the pigtail catheter. The patient did not require haemodialysis. Because of infection identified within the urinary tract, an antibiotic was introduced. The patient also received 2,000 units of erythropoietin (Eprex ${ }^{\circledR}$ ) once a week. The function of the transplanted kidney was stabilized. The patient was discharged home with normal laboratory parameters (Table 1). Other examinations (bone scintigraphy, chest radiography) revealed no progression of the disease.

Eight years postoperatively, the function of the kidney is normal, with a moderate dilatation of the pyelocalyceal system, and urea and creatinine concentration in the blood serum amounting to $2.3 \mathrm{mg} / \mathrm{dl}$ and $82 \mathrm{mg} / \mathrm{dl}$, respectively. The GFR is $33 \mathrm{ml} / \mathrm{min} / 1.73 \mathrm{~m}^{2}$.

\section{Discussion}

Nephron sparing surgery (NSS) techniques are fullyfledged procedures comparable to radical nephrectomy in the treatment of renal carcinoma, particularly in patients with a single kidney, bilateral renal tumours and impaired kidney function, and patients with tumours below $4 \mathrm{~cm}$ in 
diameter and correct renal function [2, 3, 8]. In the initial period, the main argument raised against NSS was a higher rate of complications relative to radical nephrectomy. Possible complications include haemorrhaging, urinary leakage, urinary fistula, renal artery thrombosis and acute renal failure [9-12]. Stephensen et al. demonstrated, however, that NSS and RN techniques had similar complication rates [13-15].

Other studies also confirmed comparable survival times in patients after NSS and after RN surgery $[3,7,9,16]$. The greatest benefit of NSS is preservation of kidney function. The risk of recurrence remains the most severe disadvantage of NSS, mainly due to occult multifocality undetected intraoperatively. The incidence of recurrence ranges between 0 and $10 \%$, and it is the lowest when the tumour has a low malignant potential and does not exceed $4 \mathrm{~cm}$ in diameter $[16,17]$. The problem of leaving a safe margin in the NSS procedure remains open to debate. If the margin is too wide, despite higher oncological safety, it may compromise renal function. The latest research confirms that the width of the resection margin is of no real significance as long as minimum "oncological cleanliness" is ensured [18, 8, 10].

Borderline cases, such as the one discussed in this article, concerning a patient with a single kidney, suffering from renal carcinoma with the tumour size exceeding $5 \mathrm{~cm}$ and infiltrating Gerota's posterior fascia and the pyelocalyceal system, need to be addressed on an individual basis. It is important, however, that the duration of warm ischaemia of the kidney does not exceed 30 minutes. For prolonged ischaemia, local hypothermia with ice or kidney perfusion with cold solution is employed to reduce damage to the renal parenchyma. Lowering the temperature of the kidney to $15-20^{\circ} \mathrm{C}$ extends the acceptable period of ischaemia up to 3 hours without any risk of parenchymal damage. Some sources claim that perfusion with cold Custodiol (histidinetryptophan-ketoglutarate - HTK) may improve late results of ex vivo kidney sparing surgery [19].

What our case demonstrates is that in spite of technically challenging conditions, ex vivo resection followed by autotransplantation is a correct solution that allows longterm functioning of the organ and patient's survival and helps avoid renal replacement therapy (haemodialysis), which is debilitating to the patients. The experience of medical centres in operations of this type is limited and it is difficult to present an objective analysis of late outcomes. These are, usually, isolated procedures or operations performed in groups of several people [20, 21-24]. Such borderline cases are usually conditional on the operator's experience.

\section{References}

1. European Network of Cancer Registries. Eurocim version 4.0. European incidence database V2.3, 730 entity dictionary (2001). Lyon 2001.

2. Ljungberg B, Cowan N, Hanbury DC, et al. Guidelines on Renal Cell Carcinoma http://www.uroweb.org/gls/pdf/Renal\%20Cell\%20Carcinoma\%202010.pdf

3. Weight CJ, Larson BT, Gao T, et al. Elective partial nephrectomy in patients with clinical T1b renal tumors is associated with improved overall survival. Urology 2010; 76: 631-7.
4. Wein AJ, Kavoussi LR, Novick AC, Partin AW, Craig A. Campbell-Walsh Urology. Peters Publisher: Saunders, Philadelphia 2007.

5. Patard JJ, Shavarts O, Lam JS, et al. Safety and efficacy of partial nephrectomy for all T1 tumors based on an international multicenter experience. J Urol 2004; 171: 2181-5.

6. Leibovich BC, Blute ML, Cheville JC, Lohse CM, Weaver AL, Zincke H. Nephron sparing surgery for appropriately selected renal cell carcinoma between 4 and $7 \mathrm{~cm}$ results in outcome similar to radical nephrectomy. J Urol 2004; 171: 1066-70.

7. Thompson RH, Siddiqui S, Lohse CM, Leibovich BC, Russo P, Blute $M L$. Partial versus radical nephrectomy for 4 to $7 \mathrm{~cm}$ renal cortical tumors. J Urol 2009; 182: 2601-6.

8. Li Q-L, Guan H-W, Zhang Q-P, Zhang L-Z, Fa-Peng Wang F-P, Liu Y-L. Optimal margin in nephron-sparing surgery for renal call carcinoma $4 \mathrm{~cm}$ or less. Eur Urol 2003; 44: 448-51.

9. Schlichter A, Wunderlich H, Junker K, Kosmehl H, Zermann DH, Schubert J. Where are the limits of elective nephron-sparing surgery in renal cell carcinoma? Eur Urol 2000; 37: 517-20.

10. Van Poppel $\mathrm{H}$. The optimal margins in nephron sparing surgery. Curr Opin Urol 2004; 14: 227-8.

11. Sutherland SE, Resnick MI, Maclennan GT, Goldman HB. Does the size of surgical margin in partial nephrectomy for renal cell carcinoma really matter? J Urol 2002; 167: 61-4.

12. Stephenson AJ, Hakimi AA, Snyder ME, Russo P. Complications of radical and partial nephrectomy in a large contemporary cohort. J Urol 2004; 171: 130-4.

13. Van Poppel H, Bamelis B, Oyen R, Baert L. Partial nephrectomy for renal cell carinoma can achieve long-term tumor control. J Urol 1998; 160: 674-8.

14. Van Poppel H, Dilen K, Baert L. Incidental renal carcinoma and nephron sparing surgery. Curr Opin Urol 2001; 11: 281-6.

15. Filipas D, Fichtner J, Spix C, Black P, Carus W, Hohenfellner R, Thüroff JW. Nephron-sparing surgery of renal cell carcinoma with a normal opposite kidney: long-term outcome in 180 patients. Urology 2000; 156: 387-92.

16. Peycelon M, Hupertan V, Comperat E, et al. Long-term outcomes after nephron sparing surgery for renal cell carcinoma larger than $4 \mathrm{~cm}$. J Urol 2008; 181: 35-41.

17. Uzzo RG, Novick AC. Nephron sparing surgery for renal tumors: indications, techniques and outcomes. J Urol 2001; 166: 6-18.

18. Lang $\mathrm{H}$, Lindner $\mathrm{V}$, Martin M, et al. Prognostic value of multifocality on progression and survival in localized renal cell carcinoma. Eur Urol 2004; 45: 749-753.

19. Geschwend JE, de Petriconi G, Maier S, Kleinschmidt K, Hautmann C. Continuous in situ cold perfusion with Histidine Tryptophan Ketoglutarate solution in nephron sparing surgery for renal tumors. J Urol 2004; 154: 1307-11.

20. Albani JM, Novick AC. Renal pseudoaneurysm after partial nephrectomy: three case reports and a literature review. Urology 2003; 62: 227-31.

21. Smith RB, de Kernion JB, Ehrlich RM, et al. Bilateral renal cell carcinoma and renal cell carcinoma in the solitary kidney. J Urol 1984; 132: 450-4.

22. Ecke M, Langen L. Partial kidney resection in kidney tumors - indications and results. Z Urol Nephrol 1989; 82: 173-9.

23. Ghavamian R, Cheville JC, Lohse CM, Weaver AL, Zincke H, Blute ML. Renal cell carcinoma in the solitary kidney: an analysis of complications and outcome after nephron sparing surgery. J Urol 2002; 168: 454-9.

24. Boutemy F, Demailly M, Petit J. Multifocal urothelial tumors and renal autotransplantation. Prog Urol 2002; 12: 1294-6.

\section{Address for correspondence}

\section{Marek Karczewsk}

Transplantology and Surgery Unit

of the Provincial Hospital in Poznań

ul. Juraszów 7/19

60-479 Poznań

tel. +48618212327

e-mail: drkarczewski@gmail.com 